\title{
A Quitting Game Framework for Self-Organized D2D Mobile Relaying in $5 \mathrm{G}$
}

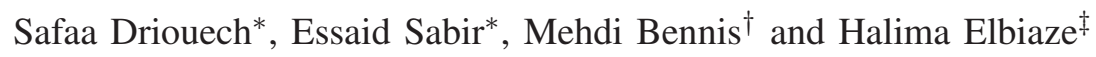 \\ *NEST Research Group, LRI lab., ENSEM, Hassan II University of Casablanca, Morocco \\ ${ }^{\dagger}$ Centre for Wireless Communications, University of Oulu, Finland \\ $\ddagger$ Computer Science department research group, University of Quebec at Montreal, Quebec, Canada \\ driouech.safaa@hotmail.fr, e.sabir@ensem.ac.ma, bennis@ee.oulu.fi, elbiaze.halima@uqam.ca
}

\begin{abstract}
Offloading the network, minimizing the power consumption as well as reducing interference are important issues in wireless networks. These requirements mandate that future cellular networks need to use Deviceto-Device communication as a key enabler. To harness this solution, we propose a two-device system that combines cellular and Device-to-Device (D2D) communication in an uplink communication. We model this system as a quitting game where devices choose simultaneously either to continue or to quit transmitting over the cellular network. The devices will strategically choose whether to compete or to cooperate through mobile relaying. We first calculate the throughput and the outage probability in a fading channel. Then we find the Sub-game Perfect Equilibrium of this game by determining the pure and mixed Nash equilibrium of each subgame. Results show that the outage probability depends on the transmission power and the distance separating a device from its serving BS. The quitting decision of devices depends on the fraction of throughput they would get after quitting, on the quitting frame and on the quitting regret.
\end{abstract}

keywords: D2D, Throughput, Self-Organized 5G, Mobile Relaying,Cooperation, Outage probability, Quitting game, Sub-game Perfect Equilibrium

\section{INTRODUCTION}

$5 \mathrm{G}$, which denotes the coming fifth generation wireless broadband technology, is not only an evolution of current network generations like smart phones, but, more significantly, it is about novel use-cases and features as well as a revolution in the Information and Communications Technology (ICT) field. One of the expectations of $5 \mathrm{G}$ is the support of dense and ultra-dense networks, which requires self-organized capability [3]. This work deals with the problem of self-organized networks allowing to strategically select the best Radio Access Network (RAN) by means of a quitting game formulation. The devices' choices might allow them to improve their throughput by minimizing the power consumption, offloading the network and reducing interference.

Communication between devices can be set up either through a base station (cellular communication) or directly between these devices, through Device-to-Device (D2D) communication. D2D communication is defined as a technology that allows devices to cooperate between each other in order to share and distribute contents directly without traversing the base station (BS), core network or access points. Furthermore, it is one of the key technologies that are supporting $5 \mathrm{G}$ architecture and that greatly increases the spectral efficiency of the network and solves poor coverage conditions, as well as improving throughput, energy efficiency, capacity, and reducing communication delay and power consumption [1]. Also, D2D offers the opportunity of mobile relaying in case the device used is in a disconnected area or in a disaster. Moreover, D2D communication can use licensed spectrum (i.e., inband) or unlicensed spectrum (i.e., outband)[2].

\section{System Model}

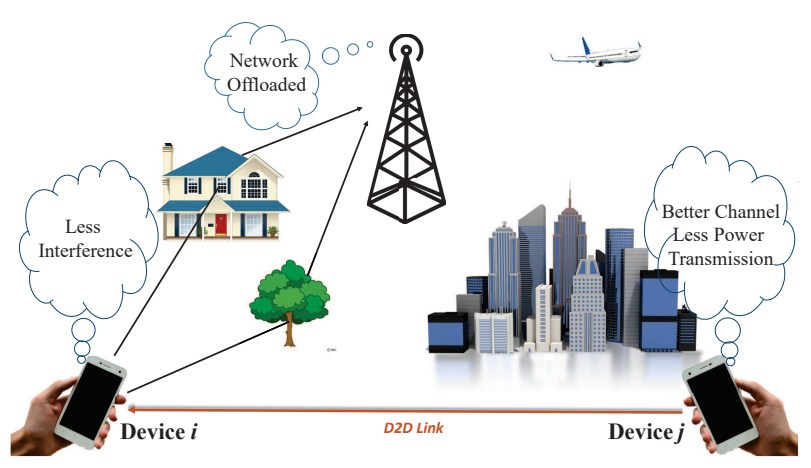

Fig. 1. Cellular/D2D communication in a fading channel

In recent years, cooperative communication with mobile relay technique has been widely used to assist the connection establishment among devices, to increase the performance of cellular users, to offload the network, reduce the energy consumption of mobile devices, enhance the quality of data transmission and help improve the capacity of the system [4]. This technique can further provide enhanced coverage, throughput, high spatial diversity and power/battery life, especially when the propagation environment experiences significant shadowing as in our case study. Mobile relaying refers to helping source node to transmit data to other nodes, thus assisting the communication between a source and multiple destinations/users. However, it is important to intelligently select the optimal and reasonable relay [4] [5] to fully benefit from cooperative communication in terms of higher transmission data rate and lower outage probability. Also, frequent relay switching can be avoided [7]. Moreover, incentives need to be provided to mobile relays to encourage them to cooperate [6].

In the above mentioned papers, the authors showed the benefits of D2D and how to efficiently choose a relay but none of them gave the optimal conditions so as to strategically 
switch from cellular communication to D2D communication. To achieve the above mentioned objectives, we use a quitting game (or war of attrition) to model the studied system. A quitting game refers to a game in which players can choose to remain in the competition or irreversibly quit [9]. The aim behind using this game is to help the devices to strategically select the best choice of either staying in the competition or cooperating.

Subgame Perfect Nash Equilibrium (SPNE) is a strategy profile that could be considered as a refinement of Nash equilibrium, due to the fact that it induces a Nash equilibrium on every subgame. Note that a common method for determining subgame perfect equilibria in the case of a finite game is backward induction [10] [11].

Our contribution consists of calculating the throughput in a fading channel, modeling the competition/cooperation (i.e. Cellular/D2D) as a quitting game and finding the Subgame Perfect Nash Equilibria. The rest of this paper is organized as follows. The system model is presented in section II. The quitting game as well as the subgame model are delineated in section III. In section IV, we detail the equilibrium analysis and numerical results are presented in section V. Finally, section VI concludes the paper and exposes our future work.

We consider a two-device system in an uplink cellular communication, where both devices are communicating with a base station through a fading channel. As this communication leads to cellular interference that each device applies to the other one, the performance of the wireless communication system is then reduced. Network congestion and fading channel are also other potential factors for performance deterioration. However, since the environment's fading is unpredictable and dynamic, we will then react on interference and congestion effect through switching the devices from the competition mode to the cooperation mode. In other words, instead that both devices communicate with the BS, one device will stay connected on cellular mode, while the other one will rely on the first one through D2D communication as Fig. 1 shows. In this way, the first device (i.e. the one that is using the cellular communication) will get rid of cellular interference, only D2D interference remains. The second device will transmit with minimum of power (i.e. D2D power) and also will dispose of the wireless fading channel since we assumed that the D2D channel is stationary, thereby offloading the network.

In our study, we represent the competition/cooperation between the two devices as a quitting game, in which each device has two choices; either to continue competing or to quit the competition. The device that quits will rely on the winner device through D2D communication to transmit its data. Thus, the winner will be considered as a mobile relay and the devices will switch from competition mode to cooperation with mutual benefits. Moreover, the longer the devices are competing they will be consuming more energy. This is due essentially to interference applied from the opponent device and cellular power consumption. Hence, it is better for one device to quit earlier and the other one to continue then to help the first one through D2D link.

\section{A. Throughput \& Outage Probability}

The throughput (also called the goodput) represents the rate of successful information bits that are delivered to the destination over a communication channel, per unit time [12]. The amount of data considered excludes protocol overhead bits as well as retransmitted data packets. The throughput of a communication system may be affected by various factors, including bit errors caused by bad status of the communication channel, problems at Multiple Access Control (MAC) level, noise and interference in the environment, proximity to the BS and Encryption/Decryption, etc. Note that high throughput and energy efficiency are among the requirements that $5 \mathrm{G}$ is expecting to improve. In our study, we consider that the throughput is affected by the opponent interference as well as the fading channel.

Based on that, we calculate the average throughput as follows:

$$
\Theta^{\prime}(\gamma)=\frac{\Theta(\gamma)}{n}(\text { bits } / s)
$$

where,

$$
\Theta(\gamma)=\frac{M}{L} R\left(1-P_{\text {out }}(\gamma)\right)=\rho\left(1-P_{\text {out }}(\gamma)\right) .
$$

Where $\rho=\frac{M}{L} R, n$ denotes the number of frames required to transmit a data. $M$ is the data payload length. $L$ denotes the packet length and $H$ is the packet header length. $R$ denotes the transmission rate and $P_{\text {out }}$ denotes the probability of outage, i.e. the probability that the SINR is less than a given SINR threshold $\left(\gamma_{t h}\right)$.

$$
P_{\text {out }}^{i}=\operatorname{Pr}\left(S I N R_{i} \leq \gamma_{t h}\right),
$$

where: $S I N R_{i}=\frac{P_{i}\left|h_{i}\right|^{2} d_{i}^{-\alpha}}{\sigma_{N}^{2}+P_{j}\left|h_{j}\right|^{2} d_{j}^{-\alpha}}$.

where $\sigma_{N}^{2}$ denotes the variance of the thermal additive white Gaussian noise. $h_{i}$ and $h_{j}$ are device $i$ and device $j$ fading channel magnitude, respectively. $d_{i}$ and $d_{j}$ are the distances that separate device $i$ and device $j$ from their serving base station, respectively. $\alpha$ is the path-loss exponent. $P_{i}$ and $P_{j}$ are the transmit powers for device $i$ and device $j$, respectively.

The outage probability of device $i$ is given by:

$$
\begin{aligned}
P_{\text {out }}^{i}= & \operatorname{Pr}\left(\frac{P_{i}\left|h_{i}\right|^{2} d_{i}^{-\alpha}}{\sigma_{N}^{2}+P_{j}\left|h_{j}\right|^{2} d_{j}^{-\alpha}} \leq \gamma_{t h}\right) \\
& =\operatorname{Pr}\left(\left|h_{i}\right| \leq \sqrt{\frac{\gamma_{t h} \sigma_{N}^{2}}{P_{i} d_{i}^{-\alpha}}+\left|h_{j}\right|^{2} \frac{\gamma_{t h} P_{j} d_{j}^{-\alpha}}{P_{i} d_{i}^{-\alpha}}}\right) \\
& =\int_{0}^{+\infty} \int_{0}^{\sqrt{\frac{\gamma_{t h} \sigma_{N}^{2}}{P_{i} d_{i}^{-\alpha}}+\left|h_{j}\right|^{2} \frac{\gamma_{t h} P_{j} d_{j}^{-\alpha}}{P_{i} d_{i}^{-\alpha}}}} f\left(h_{i}\right) f\left(h_{j}\right) \mathrm{d} h_{i} \mathrm{~d} h_{j}
\end{aligned}
$$

Notice that $h_{i}$ and $h_{j}$ are two Gaussian Random Variables with mean zero and variance $\sigma_{i}^{2}$ and $\sigma_{j}^{2}$, respectively. They follow a Rayleigh distribution with density function $f(\cdot)$ given by

$$
f(h, \sigma)=\frac{h}{\sigma^{2}} e^{-\frac{h^{2}}{2 \sigma^{2}}} .
$$

Therefore, the outage probability can be expressed as follows:

$$
P_{\text {out }}^{i}=1-\frac{P_{i} d_{i}^{-\alpha} \sigma_{i}^{2}}{\gamma_{t h} P_{j} d_{j}^{-\alpha} \sigma_{j}^{2}+P_{i} d_{i}^{-\alpha} \sigma_{i}^{2}} e^{-\frac{\gamma_{t h} \sigma_{N}^{2}}{2 P_{i} d_{i}^{-\alpha} \sigma_{i}^{2}}}
$$

\section{Quitting GAme}

Quitting game is a game in which players can choose to remain in the competition or irreversibly quit. Existing works dealing with quitting games mechanism consider simple noncooperative stochastic games with a finite number of players, where each player has only two possible actions, continue 


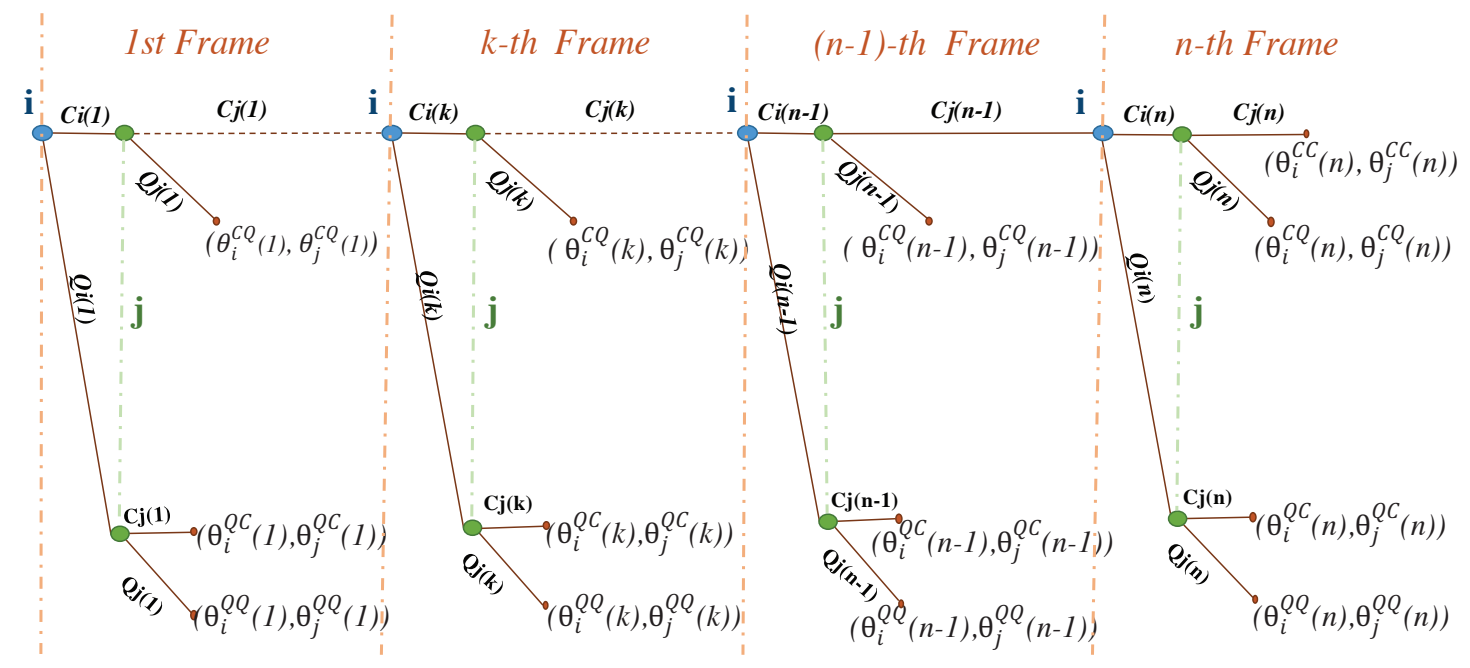

Fig. 2. Extensive form of the cellular quitting game

and quit. All players make their decisions simultaneously and independently of each other. Depending on the ultimate goal, the game ends when at least one player chooses to quit as in [14], or as soon as there is only a single player left [15].

To explicitly clarify the studied game, a game tree illustrated by Fig. 2 is showing the interactions between the players and the order of events that take place after each decision. Each decision is made at a node and the payoffs are written at the end of the tree's branches. The game is involving two players (devices) $P_{i}$ (Player $i$ ) and $P_{j}$ (Player $j$ ) and each player in each frame chooses, simultaneously, whether to Compete $\langle\mathrm{C} »$ or to Quit $《 \mathrm{Q} »$. Payoffs are represented by $\Theta_{i}^{C C}$ and $\Theta_{j}^{C C}$ denoting the throughput of device $i$ and device $j$ respectively, if they both decide to continue. If device $i$ continues while device $j$ quits, their respective throughput payoffs are $\Theta_{i}^{C Q}$ and $\Theta_{j}^{C Q}$ while if device $i$ quits and device $j$ continues they will have $\Theta_{i}^{Q C}$ and $\Theta_{j}^{Q C}$ as throughputs, respectively. Finally, if they both quit they will have $\Theta_{i}^{Q Q}$ and $\Theta_{j}^{Q Q}$ as throughput payoffs.

In order to study the behavior of both devices in each part of the game, we will (i) analyze the sub game perfect Nash equilibrium, (ii) find the Pure and Mixed Nash equilibrium in each sub-game and then (iii) conclude the Sub-game Perfect Nash Equilibria (SPNE). To do so, we will use the backward induction to solve the finite game tree.

\section{A. Sub-game Model}

The considered game contains $n$ sub-games as shown in Fig. 2. A sub-game is a set of choices within a game tree that also contain well-defined game tree themselves. The backward induction of the sub-game starts from the last frame and continues to the first frame, in every sub-game (i.e. frame) every device chooses simultaneously whether to continue competing or to quit. If both devices decide to continue, they will both stay connected to the BS to transmit their data through cellular communication, in this case each of the devices will suffer from interference applied from its opponent and they will transmit with a high power. However, if one device quits, the game ends, and it will rely on the other device, which decided to continue, through D2D link. In this case, the device that quits will transmit with less power while the other device will get rid of interference and the network will be offloaded. If both devices quit at once, they will not complete transmitting their data, so the aim of this communication will not be satisfied, which will lead to a regret from both devices. Overall, we considered this n-frame game as a repeated game with memory that ends as soon as one device quits.

In order to understand this sub-game, we present its strategic form in Table III-A, that contains the throughput (utility) that each device will own on the $k$-th frame, depending on its choice.

\begin{tabular}{|c|c|c|c|}
\hline \multirow{4}{*}{ Device $i$} & \multirow{4}{*}{$\begin{array}{c}\text { Continue } \\
\text { Quit }\end{array}$} & \multicolumn{2}{|c|}{ Device $j$} \\
\hline & & Continue & Quit \\
\hline & & $\Theta_{i}^{C C}(\mathrm{k}), \Theta_{j}^{C C}(\mathrm{k})$ & $\Theta_{i}^{C Q}(\mathrm{k}), \Theta_{j}^{C Q}(\mathrm{k})$ \\
\hline & & $\Theta_{i}^{Q C C}(\mathrm{k}), \Theta_{j}^{Q C}(\mathrm{k})$ & $\Theta_{i}^{Q Q}(\mathrm{k}), \Theta_{j}^{Q Q}(\mathrm{k})$ \\
\hline
\end{tabular}

The throughput in each case will be expressed as follows: (Continue, Continue): When both devices will decide to continue until getting their services from the base station, they will have $\Theta_{i}^{C C}(\mathrm{k})$ and $\Theta_{j}^{C C}(\mathrm{k})$ as throughput for device $i$ and device $j$, respectively.

$$
\begin{gathered}
\left.\Theta_{i}^{C C}(k)=(k-1)\right) \rho_{i}\left(1-P_{\text {out }, c}^{i}\right)+C_{p}^{i} \\
\Theta_{j}^{C C}(k)=(k-1) \rho_{j}\left(1-P_{\text {out }, c}^{j}\right)+C_{p}^{j}
\end{gathered}
$$

Note that $C_{p}^{i}$ and $C_{p}^{j}$ are the continuation payoffs of device $i$ and $j$ that depends on the future decision of both devices if they decide to continue competing, respectively.

(Continue, Quit): When device $j$ quits and relies on device $i$ that continues:

$$
\Theta_{i}^{C Q}(k)=(k-1) \rho_{i}\left(1-P_{\text {out }, c}^{i}\right)+(n-k+1) x_{i} \rho_{i}\left(1-P_{\text {out }, C Q}^{i}\right)
$$

$\Theta_{j}^{C Q}(k)=(k-1) \rho_{j}\left(1-P_{\text {out }, c}^{j}\right)+(n-k+1)\left(1-x_{i}\right) \rho_{i}\left(1-P_{\text {out }, C Q}^{i}\right)$

(Quit, Continue): When device $i$ quits and relies on device $j$ that continues:

$\Theta_{i}^{Q C}(k)=(k-1) \rho_{i}\left(1-P_{\text {out }, c}^{i}\right)+(n-k+1)\left(1-x_{j}\right) \rho_{j}\left(1-P_{\text {out }, Q C}^{j}\right)$ 
$\Theta_{j}^{Q C}(k)=(k-1) \rho_{j}\left(1-P_{\text {out }, c}^{j}\right)+(n-k+1) x_{j} \rho_{j}\left(1-P_{\text {out }, Q C}^{j}\right)$

(Quit, Quit): When both devices quit at the same frame:

$$
\begin{aligned}
& \Theta_{i}^{Q Q}(k)=(k-1) \rho_{i}\left(1-P_{\text {out }, c}^{i}\right)-(n-k+1) r_{i} \\
& \Theta_{j}^{Q Q}(k)=(k-1) \rho_{j}\left(1-P_{\text {out }, c}^{j}\right)-(n-k+1) r_{j}
\end{aligned}
$$

Where $r_{i}$ and $r_{j}$ are regrets that both devices will earn because of quitting at once, thus not achieving their transmissions.

\section{EQUILIBRIUM ANALYSIS}

In this section, we aim to find the equilibrium strategies of each player (i.e. device $i$ and device $j$ ). Different from our previous work where we solved the entire equilibrium of the game [9], in this work we are interested in looking for the equilibrium in each part of the game, and also the probability upon whether each device could either continue or quit in each subgame. We will start by deriving the Pure Nash Equilibrium (PNE), then we will determine the Mixed Nash Equilibrium (MNE) through calculating the continuing and quitting probability of both devices.

\section{A. Sub-game Perfect Equilibria}

In game theory, a strategy could be considered as a subgame perfect equilibrium if it represents a Nash equilibrium of every subgame of the original game. In other words, the subgame perfect Nash equilibrium is found by treating every part of the game as a One-Shot game and finding the best-response (strategy) of each sub-game. Thus, the set of subgame perfect equilibria for a given game will always be a subset of the set of Nash equilibria for that game [16].

A common way for finding subgame perfect equilibria of a finite game is "backward induction". Generally, it first consists of considering the last actions of the game and determines which actions the final player should take to maximize its utility. One then supposes that the last actor will do these actions, and considers the second to last actions, again choosing those that maximize that actor's utility. This process continues until one reaches the first move of the game. In other words, the analysis is done from the bottom of the game to the top. The strategies which remain are the set of all subgame perfect equilibria of the game.

In our case, both players will choose simultaneously the strategy that will maximize their utilities, that is to say, their choices will depend on the future action that represents a Nash equilibrium (best response) of the next sub-game. To explain more, by seeing the tree on Fig. 2, the backward induction can be defined as the act of eliminating branches which would involve any player making a move that is not credible (because it is not optimal) from that node.

Now we're going to consider our quitting game as $n$ subgames and determine the Pure and Mixed Nash equilibrium of each sub-game and then deduce the subgame perfect equilibrium of the whole game.

\section{B. Pure Nash Equilibrium}

In such a non cooperative sub-game, a set of strategies are in Pure Nash Equilibrium (PNE) when no player has an incentive to unilaterally change or improve his strategy given every other player's strategy. Applying backwards induction to this game allowed us to find the number of equilibria of each sub-game depending on $k$ and $h$, the frame they are playing in and the number of frames after which a device will decide to quit, respectively. Generally, in the two-player war of attrition game, it is an equilibrium for one player to exit immediately at the beginning of the game (zero effort) and for the other player to never exit until finishing its transmission; in this way no sources are "wasted" (i.e. zero fighting costs) [15]. Therefore, the Nash equilibrium solutions of this noncooperative quitting game are derived in the following:

Lemma 1. In the $k$-th frame, the (continue, continue) case will have $2(n-k)+1$ possible equations (continuation payoffs) and there will be $6(n-k)+3$ possible PNEs under conditions.

Proof. The continuation payoffs $\left(C_{p}\right)$ depend on the decision of next frames. In addition, by applying the backward induction, we figured out that in each frame there are three possible PNEs under conditions, which are (Continue, Continue), (Continue,Quit) and (Quit,Continue). Moreover, the application of the backward induction leads to the addition of two more $C_{p}$, that contains the conditions on the added frame on either (Continue,Quit) or (Quit,Continue), in each time we go back of one frame. The main idea is to find the PNE in the last sub-game and roll back theses possible payoffs to the previous frame.

Through the backward induction, we suppose that in the $(n-t)^{t h}$ frame we have $2 t+1$ possible equations for the (Continue,Continue) case and $6 t+3$ PNE under conditions. And knowing that in each time we go back of one frame two other $C_{p}$ are added, so the $(n-(t+1))^{t h}$ frame will have: $(2 t+1)+2=2 t+3$ equations (i.e. possibilities) for the (Continue, Continue) case. Assuming that in each possibility, three PNE under conditions are found : (Continue,Continue), (Continue, Quit) and (Quit,Continue). So the total PNE under conditions are:

$3(2 t+3)=3(2 t+2+1)=3(2(t+1))+1)=6(t+1)+3$,

which proves by recurrence the above lemma. By changing variables such that $k=n$ - $t, k \in\{1, \ldots, n-1\}$, the PNE under conditions of the $(k-1)-t h$ frame are: $6(n-(k-1))+3$.

Once the devices are in the $k^{\text {th }}$ frame, they will either both continue competing (Continue, Continue), one device will continue while the other one will quit (Continue, Quit) or (Quit, Continue), or both of them will quit the competition. Moreover, if the devices will decide to continue competing in the current frame, they will either both continue competing until the end (i.e. $n^{\text {th }}$ frame), one device will continue competing while the other one will quit after $h$ frames, or they will both quit after $h$ frames. Note that $h \in\{1, . .,(n-k)\}$.

Lemma 2. The actions (Continue, Continue), (Continue, Quit) and (Quit, Continue) are the Perfect Sub-game Nash Equilibria, under conditions, of this quitting game.

Lemma 3. If the devices decide to continue competing until the end, the PNEs will be as follows:

(Continue, Continue) could be a PNE if:

$$
\rho_{i}\left(1-P_{o u t, c}^{i}\right) \geq\left(1-x_{j}\right) \rho_{j}\left(1-P_{\text {out }, Q C}^{j}\right)
$$

$\&$

$$
\rho_{j}\left(1-P_{\text {out }, c}^{j}\right) \geq\left(1-x_{i}\right) \rho_{i}\left(1-P r_{\text {out }, C Q}^{i}\right)
$$

(Continue, Quit) could be a PNE if:

$$
x_{i} \rho_{i}\left(1-P_{\text {out }, C Q}^{i}\right) \geq-r_{i}
$$


$\&$

$$
\left(1-x_{i}\right) \rho_{i}\left(1-P_{\text {out }, C Q}^{i}\right) \geq \rho_{j}\left(1-P_{\text {out }, c}^{j}\right)
$$

(Quit, Continue) is a PNE if:

$$
\left(1-x_{j}\right) \rho_{j}\left(1-P_{\text {out }, Q C}^{j}\right) \geq \rho_{i}\left(1-P_{\text {out }, c}^{i}\right)
$$

$\&$

$$
x_{j} \rho_{j}\left(1-P_{\text {out }, Q C}^{j}\right) \geq-r_{j}
$$

Lemma 4. If the devices decide to play (Continue, Quit) after $h$ frames, the PNEs will be as follows:

(Continue, Continue) is a PNE if:

$$
\begin{aligned}
& h \rho_{i}\left(1-P_{\text {out }, c}^{i}\right)+((n-k)-h+1) x_{i} \rho_{i}\left(1-P_{\text {out }, C Q}^{i}\right) \geq \\
& ((n-k)+1)\left(1-x_{j}\right) \rho_{j}\left(1-P_{\text {out }, Q C}^{j}\right)
\end{aligned}
$$

$\&$

$$
\rho_{j}\left(1-P_{\text {out }, c}^{j}\right) \geq\left(1-x_{i}\right) \rho_{i}\left(1-P_{\text {out }, C Q}^{i}\right)
$$

(Continue, Quit) is a PNE if:

$$
x_{i} \rho_{i}\left(1-P_{\text {out }, C Q}^{i}\right) \geq r_{i}
$$

$\&$

$$
\left(1-x_{i}\right) \rho_{i}\left(1-P_{\text {out }, C Q}^{i}\right) \geq \rho_{j}\left(1-P_{\text {out }, c}^{j}\right)
$$

(Quit, Continue) is a PNE if:

$$
\begin{aligned}
& ((n-k)+1)\left(1-x_{j}\right) \rho_{j}\left(1-P_{\text {out }, Q C}^{j}\right) \geq h \rho_{i}\left(1-P_{\text {out }, c}^{i}\right) \\
& +((n-k)-h+1) x_{i} \rho_{i}\left(1-P_{\text {out }, C Q}^{i}\right)
\end{aligned}
$$

$\&$

$$
x_{j} \rho_{j}\left(1-P_{\text {out }, Q C}^{j}\right) \geq r_{j}
$$

Lemma 5. When the devices decide to play (Quit, Continue) after $h$ frames, the PNEs are symmetrical to those of the above case.

Proof. As stated earlier, in the $k-t h$ frame, the devices will either continue competing till the horizon, one of both will continue while the other one will quit and rely on the first one through D2D link or they will both quit and own a regret. By acting so, the throughput of device $i$ and device $j$ will be as in equations $\{7,9,11,13\}$, and $\{8,10,12,14\}$, respectively.

Furthermore, the continuation payoffs that appear in equations 7 and 8 depend on what device $i$ and device $j$ would get on the next frames, if they both continue on the current frame, whether they will continue until the end or one device will quit after $h$ frames while the other one will continue. We did not take the (Quit,Quit) case because our aim is to find the equilibrium and (Quit, Quit) is not a PNE, as it will be shown in the sequel.

1- When both devices decide to continue competing until the end:

$$
C_{p}^{y}=(n-k+1) \rho_{y}\left(1-P_{\text {out }, c}^{y}\right), \quad y=\{i, j\}
$$

2- When the devices decide to play (Continue, Quit) after $h$ frames:

$$
C_{p}^{i}(h)=h \rho_{i}\left(1-P_{o u t, c}^{i}\right)+(n-k-h+1) x_{i} \rho_{i}\left(1-P_{\text {out }, C Q}^{i}\right)
$$

$C_{p}^{j}(h)=h \rho_{j}\left(1-P_{\text {out }, c}^{j}\right)+(n-k-h+1)\left(1-x_{i}\right) \rho_{i}\left(1-P_{\text {out }, C Q}^{i}\right)$

3- When the devices decide to play (Quit, Continue) after $h$ frames:

$C_{p}^{i}(h)=h \rho_{i}\left(1-P_{\text {out }, c}^{i}\right)+(n-k-h+1)\left(1-x_{j}\right) \rho_{j}\left(1-P_{\text {out }, Q C}^{j}\right)$

$$
C_{p}^{j}(h)=h \rho_{j}\left(1-P_{\text {out }, c}^{j}\right)+(n-k-h+1) x_{j} \rho_{j}\left(1-P_{\text {out }, Q C}^{j}\right)
$$

Furthermore, we know that a PNE occurs if and only if this strategy's response (utility) is the best among all other strategies, then:

- (Continue, Continue) is a pure Nash Equilibrium iff: $\Theta_{i}^{C C}(\mathrm{k}) \geq \Theta_{i}^{Q C}(\mathrm{k})$ And $\Theta_{j}^{C C}(\mathrm{k}) \geq \Theta_{j}^{C Q}(\mathrm{k})$

- (Continue, Quit) is a pure Nash Equilibrium iff: $\Theta_{i}^{C Q}(\mathrm{k}) \geq \Theta_{i}^{Q Q}(\mathrm{k})$ And $\Theta_{j}^{C Q}(\mathrm{k}) \geq \Theta_{j}^{C C}(\mathrm{k})$

- (Quit, Continue) is a pure Nash Equilibrium iff: $\Theta_{i}^{Q C}(\mathrm{k}) \geq \Theta_{i}^{C C}(\mathrm{k})$ And $\Theta_{j}^{Q C}(\mathrm{k}) \geq \Theta_{j}^{Q Q}(\mathrm{k})$

- (Quit, Quit) is a pure Nash Equilibrium iff: $\Theta_{i}^{Q Q}(\mathrm{k}) \geq \Theta_{i}^{C Q}(\mathrm{k})$ And $\Theta_{j}^{Q Q}(\mathrm{k}) \geq \Theta_{j}^{Q C}(\mathrm{k})$

The solution of those inequalities gives the conditions in the above lemma. While for the (Quit,Quit) case, the conditions are:

$$
x_{i} \rho_{i}\left(1-P_{\text {out }, C Q}^{i}\right) \leq-r_{i} \& x_{j} \rho_{j}\left(1-P_{\text {out }, Q C}^{i}\right) \leq-r_{j}
$$

This is contradictory, due to the fact that the throughput is not negative and could not be less than a regret (which is a negative expression).

\section{Mixed Nash Equilibrium}

In a mixed strategy equilibrium both players fight for a positive expected time, and each player should be indifferent over the actions taken. In other words, in a mixed equilibrium each device will be indifferent between dropping out at $k$ or waiting to drop out at $k+1$.

The Mixed Nash Equilibrium (MNE) of each sub-game depends on the devices continuation payoffs $C_{p}$, whether they will continue or quit after $h$ frames.

Lemma 6. If the devices decide to continue competing until the end, the MNE will be as follows:

$$
\left\{\begin{array}{l}
p=\frac{1}{1+\frac{\left(1-x_{i}\right) \rho_{i}\left(1-P_{\text {out }, C Q}^{i}\right)-\rho_{j}\left(1-P_{\text {out }, c}^{j}\right)}{r_{j}+x_{j} \rho_{j}\left(1-P_{\text {out }, Q C}^{j}\right)}} \\
q=\frac{1}{1+\frac{\left(1-x_{j}\right) \rho_{j}\left(1-P_{\text {out }, Q C}^{j}\right)-\rho_{i}\left(1-P_{\text {out }, c}^{i}\right)}{r_{i}+x_{i} \rho_{i}\left(1-P_{\text {out }, C Q}^{i}\right)}}
\end{array}\right.
$$

With $p$ and $q$ are the probability of continuing of device $i$ and device $j$, respectively.

Lemma 7. If the devices decide to play (Continue, Quit) after $h$ frames, which means device $i$ will continue and device $j$ will quit, the PNE will be as follows:

$$
\left\{\begin{array}{l}
p(h)=\frac{1}{1+\frac{h\left(1-x_{i}\right) \rho_{i}\left(1-P_{o u t}^{i}, C Q\right.}{((n-k)+1)\left(r_{j}+x_{j} \rho_{j}\left(1-P_{j}^{j}\left(1-P_{\text {out }, Q C}^{j}\right)\right)\right.}} \\
q(h)=\frac{1}{1+\frac{(n-k+1)\left(1-x_{j}\right) \rho_{j}\left(1-P_{\text {out }, Q C}^{j}\right)-h \rho_{i}\left(1-P_{\text {out }, c}^{i}\right)-A}{((n-k)+1)\left(r_{i}+x_{i} \rho_{i}\left(1-P_{\text {out }, C Q}^{i}\right)\right)}}
\end{array}\right.
$$

With: $A=(n-k-h+1) x_{i} \rho_{i}\left(1-P_{o u t, C Q}^{i}\right)$

Lemma 8. The MNEs under conditions, if the devices decide to play (Quit, Continue) after $h$ frames, are symmetrical to those of the above case.

Proof. In a mixed Nash equilibrium, each player becomes just indifferent concerning the choice of his pure strategies. Let $p$ and $q$ denote the probability of continuing for device $i$ and device $j$, respectively. Then:

$$
q \theta_{i}^{C C}(k)+(1-q) \theta_{i}^{C Q}(k)=q \theta_{i}^{Q C}(k)+(1-q) \theta_{i}^{Q Q}(k)
$$




$$
p \theta_{j}^{C C}(k)+(1-p) \theta_{j}^{Q C}(k)=p \theta_{j}^{C Q}(k)+(1-p) \theta_{j}^{Q Q}(k)
$$

Which leads to the following expressions:

$$
\left\{\begin{array}{l}
p=\frac{\theta_{j}^{Q Q}(k)-\theta_{j}^{Q C}(k)}{\theta_{j}^{C C}(k)-\theta_{j}^{Q C}(k)-\theta_{j}^{C Q}(k)+\theta_{j}^{Q Q}(k)} \\
q=\frac{\theta_{i}^{Q Q}(k)-\theta_{i}^{C Q}(k)}{\theta_{i}^{C C}(k)-\theta_{i}^{Q C}(k)-\theta_{i}^{C Q}(k)+\theta_{i}^{Q Q}(k)}
\end{array}\right.
$$

Moreover, by replacing each throughput by its expression, we will have the expressions presented in the previous lemmas.

\section{NumERICAL RESUlts}

In this study, we set up the simulations using Matlab. We plot the outage probability of device i function of its transmission power, device j' $\mathrm{s}$ transmission power and the distance that separates device i from the BS. Then, we studied the case where device $j$ tends to quit and device $i$ continues until the end and cooperates with the first one using D2D mode (case 2). Note that the results of the third case are symmetrical to the second one. To do so, we took $P_{i}^{c}=P_{j}^{c}=$ $0.1 \mathrm{~W}, P_{i}^{D}=P_{j}^{D}=50 \mathrm{~mW}, R_{i}=R_{j}=1 \mathrm{Mbit} / \mathrm{s}, L=M=1024 \mathrm{bits}$, $n=50$ ( $n$ is the number of frames required for transmitting a data), $k=20, \gamma_{t h}=1, d_{i}=d_{j}=10 \mathrm{~m}, \alpha=0.2, \sigma_{i}^{2}=\sigma_{j}^{2}=0.1$ and $\sigma_{N}^{2}=0.02$.

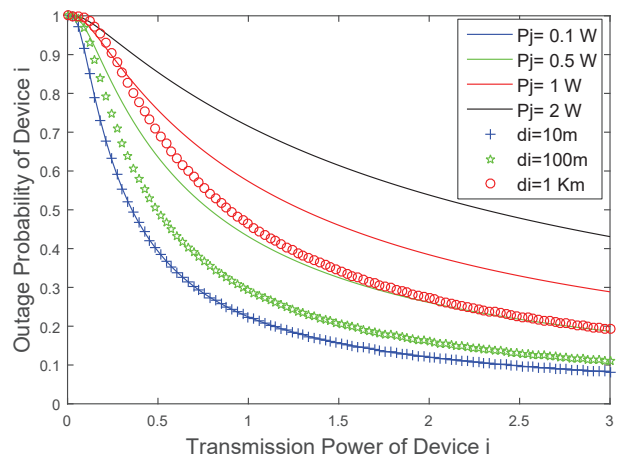

Fig. 3. Outage Probability of device i function of its transmission power

Fig. 3 shows that the outage probability of device i decreases as its transmission power increases. Whereas, it increases as device $\mathrm{j}$ transmission power increases and the distance that separates the device form its serving BS increases too. This is due to the fact that, first, interference increase as the opponent device's transmit power increases, second, the more the distance is long, the more the signal is weakened due to the path loss and fading effects.

Fig. 4 shows clearly that the probability of continuing of device $i(p)$ decreases as $h$ (i.e. the quitting frame of device $\mathbf{j}$ ) increases. In other words, if the opponent device will quit later, the device tends to quit earlier (its probability of continuing decreases), so as to have at least one device in the cellular communication in order to insure the continuity of the service. While it increases as $x_{i}$ and $x_{j}$ increase, the parts of the throughput device $\mathrm{i}$ and device $\mathrm{j}$ will use for themselves during D2D communication, respectively, as well as if $r_{j}$ increases too (i.e the regret of device $j$ decreases). That is to say, the more the fraction of throughput that the device will use for itself in D2D communication is high $\left(x_{i}\right)$ or the fraction given by the opponent device is low $\left(1-x_{j}\right)$, the more it tends to stay in the cellular communication and vice versa,
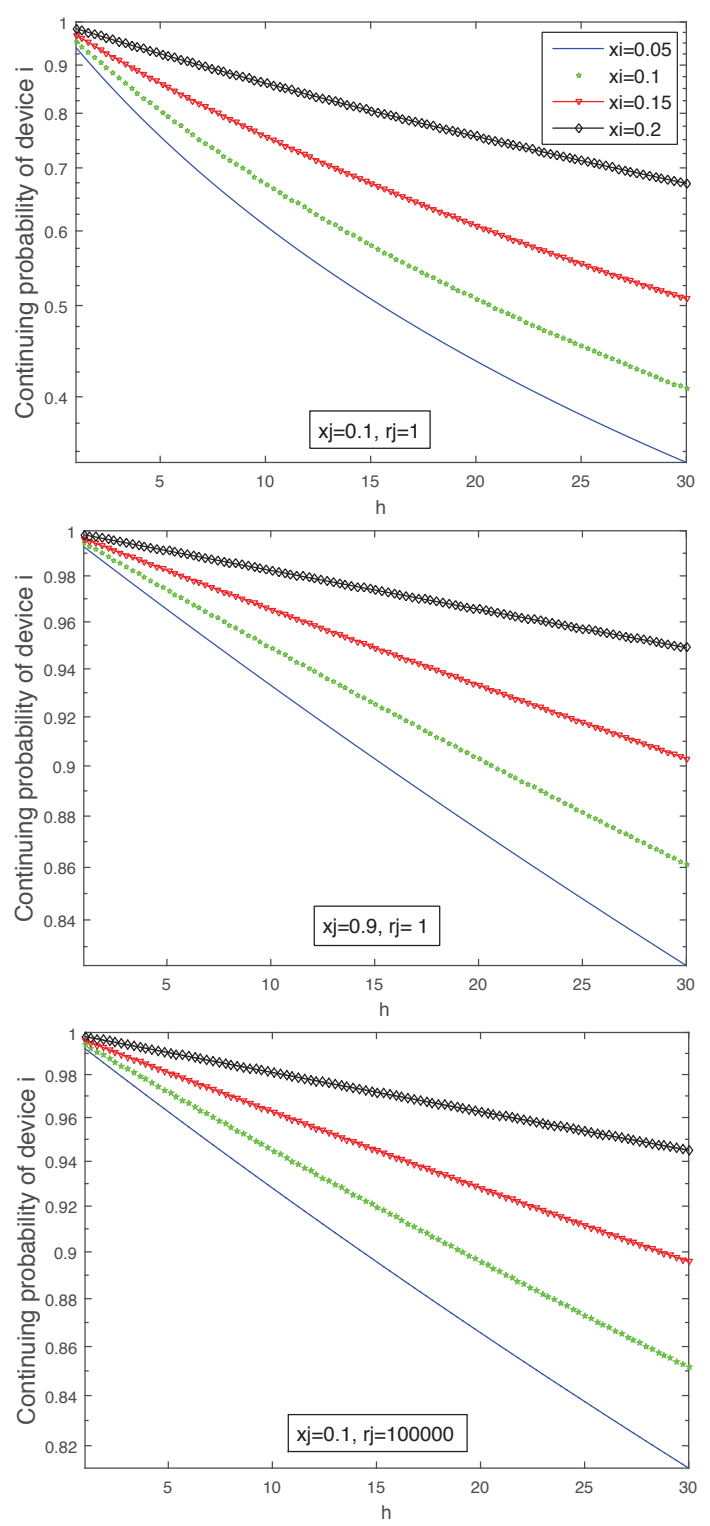

Fig. 4. Continuing Probability of device $i$ function of the quitting frame $(h)$ in the $2^{n d}$ case

because it will benefit from higher throughput than during D2D communication. Also, if the opponent device's regret of quitting decreases $\left(-r_{j}\right)$, this last will prefer to quit once weakened by interference or else, thus the device will tend to continue if the first will lean to quit.

Concerning the continuing probability of device $j(q)$ seen in Fig. 5, it increases as the parts of the throughput device $i$ and device $j$ will use for themselves during D2D communication, $x_{i}$ and $x_{j}$, increase, respectively, and as regret of device $i\left(-r_{i}\right)$ decreases. In other words, if the fraction given from device $i$ to device $j\left(1-x_{i}\right)$ during D2D, is high, device $j$ tends to quit earlier. Besides, if device $j$ will give a small fraction of its throughput it chooses better to stay in the cellular mode, then in both cases device $j$ will benefit from high throughput. Also, more $h$ is high (i.e device $\mathrm{j}$ quits later) $q$ increases, thus the more the device $j$ quitting frame $h$ is higher, the more its probability to continue competing in the $k-t h$ frame gets higher too, which is logical. Furthermore, symmetrical behaviors could be seen in the third case. 

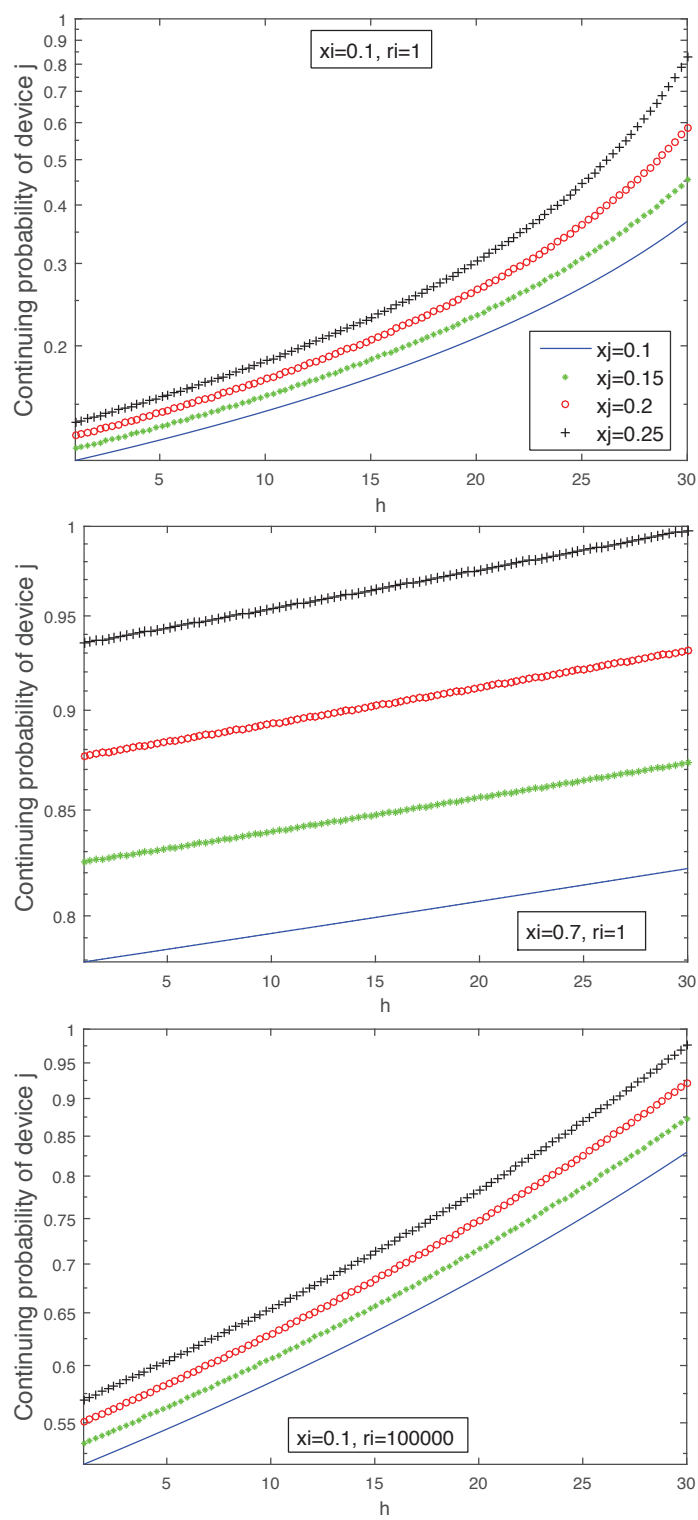

Fig. 5. Continuing Probability of device $j$ function of the quitting frame $(h)$ in the $2^{n d}$ case

The quitting decision of device $i$ in this game depends on $x_{i},\left(1-x_{j}\right)$ and $(-r)$, the fraction of throughput device $i$ will use, the fraction given by device $\mathrm{j}$ during D2D communication, and the regret of quitting the communication (in case they quit both), respectively. The device will tend to quit earlier if: first the fraction of throughput that the other device will reserve to it $\left(1-x_{j}\right)$ in D2D is high, second if instead it is asked to give a high fraction of throughput to the device that will quit $\left(x_{i}\right)$ and third if the opponent device's regret is high. As these factors act directly on the throughput of each device, they will define whether the device will transmit through cellular or D2D network, depending on the situation where the throughput will be the highest.

\section{CONCLUSiON}

Two main directions have been presented in this paper: first we calculated the probability of outage in a two-device uplink fading system, then we modeled this system as a quitting game where one device tends to quit the competition while the other one will stay connected to the BS till the horizon. The device that will quit the competition (i.e. the cellular communication) will rely on the winner (i.e. the one that stays) through a D2D link, thus the system will switch from a competition (cellular communication) to a cooperation (D2D communication). The aim of this paper is to present a self-organized D2D system as one of the $5 \mathrm{G}$ trends, to study the behavior of theses devices through using quitting game. Moreover, this strategy will lead principally to getting rid of cellular interference, offloading the network and minimizing the power consumption (which will lead to minimizing the probability of outage). Furthermore, we have relied on the backward induction to find the Pure and Mixed Subgame Perfect Equilibria of this game. Results show that the outage probability depends on the transmission power and the distance between a device and its serving BS. Moreover, a device tends to quit earlier if: first the fraction of throughput taken from its opponent increases, second, the regret of quitting of its opponent is high and also if its opponent's quitting frame increases too. Our future work will shed light on a more general and realistic scheme with several devices, where devices have to strategically choose between D2D and cellular communication so as to ensure an ultra reliable and low latency communication.

\section{REFERENCES}

[1] X.(Sherman) Shen, "Device-to-Device Communication in $5 G$ Cellular Networks", IEEE Network, vol. 29, 24 March 2015.

[2] A. Asadi, Q. Wang and V. Mancuso, "A Survey on Device-to-Device Communication in Cellular Networks" IEEE Communications Surveys \& Tutorials, Volume: 16, 1801-1819, 24 April 2014.

[3] E.Hossain and M.Hasan, "5G Cellular: Key Enabling Technologies and Research Challenges" IEEE Instrumentation \& Measurement Magazine, 11-21, 15 May 2015.

[4] Bojiang Ma, H. Shah-Mansouri and V. W.S. Wong, "Full-duplex Relaying for D2D Communication in mmWave based $5 G$ Networks", IEEE Trans. Wireless Communications, 1536-1276, 17 April 2018.

[5] M. THI, T. HUYNH and W. HWANG, "Proportional Selection of Mobile Relays in Millimeter-Wave Heterogeneous Networks", IEEE Access, volume 6, 16081-16091, 27 March 2018.

[6] B. S. Khan, S. Jangsher and F. Bhatti, "Profitable Relay Selection in Cooperative Cellular Network with Mobile Relays", IEEE 86th Vehicular Technology Conference, 24-27 Sept. 2017.

[7] D. Xiao, W. Xuanli, M. Chuiyang, H. Shuai and F. Xiaojie, "A Mobile Relay Selection Strategy in Cooperative Spectrum Sharing Framework", IEEE ICC Ad-Hoc and Sensor Networking Symposium, 21-25 May 2017, Paris, France.

[8] H. Singh and A. Singh Sappal, "Analysis of Rayleigh Fading Channel in FSO System with respect to Eb/NO for Different Modulation Schemes" IEEE WiSPNET conference, 22-24 March 2017, Chennai, India.

[9] S. Driouech, E. Sabir and H. Tembine, "Self-Organized Device-to-Device Communications as a non-Cooperative Quitting Game", in proceeding of IEEE Wincom17 conference, November 01-04, 2017, Rabat.

[10] B. W. Abegaz and Dr. Satish M. Mahajan "Optimal Dispatch Control of Energy Storage Systems using Forward-Backward Induction" IEEE Clean Electrical Power (ICCEP), International Conference, 731-736, 1618 June 2015, Taormina, Italy.

[11] El Karoui.N., Quenez.M.C. "Non-Linear Pricing Theory and Backward Stochastic Differential Equations" Part of the Lecture Notes in Mathematics book series (LNM), volume 1656, Springer, Berlin, Heidelberg.

[12] F.Meshkati, H. V. Poor, S.C. Schwartz and N.B. Mandayam, "An Energy-Efficient Approach to Power Control and Receiver Design in Wireless Data Networks" IEEE Transactions on Communications, Volume: 53, 1885-1894, 14 November 2005.

[13] K. Fischer, "e-equilibria in quitting games-basics" Published in arXiv, Conference proceedings of the "International Symposium on Dynamic Games and Applications", 12 Jan 2011.

[14] D. P.Myatt, "Instant Exit From The War of Attrition" , Nuffield college, University of Oxford, October 27, 2003.

[15] J. Levin, "Wars of Attrition" , Originally written by Jeremy Bulow, October 2004.

[16] Y. Zhai and Q. Zhao, "Oligopoly Dynamic Pricing: A Repeated Game with Incomplete Information" IEEE ICASSP International Conference, 4772-4775, 20-25 March 2016, Shanghai, China. 